\section{PRINCIPLES AND PRACTICE OF INTENSIVE CARE MONITORING}

Martin J. Tobin (ed), The McGraw-Hill Co., New York, 1998, ISBN 0-07-065034-2, 1525 pages, US\$145.00

This book is a monumental opus covering every aspect of intensive care monitoring. The introductory section covers general monitoring principles and is followed by three sections that extensively review basic respiratory, cardiovascular, and neurologic organ systems. Four additional sections are dedicated to (1) monitoring secondary organ systems, (2) special situations, such as patient monitoring during transportation, (3) computers in monitoring, and (4) ethical-legal issues.

Because of the complexity of modern monitoring devices in the intensive care unit, many artifacts are introduced that may interfere with clinical interpretation. The first section reviews the features of reliable monitoring signals. The basic principles of phsics, necessary to corrrectly interpret unreliable signals, are analyzed in detail by experienced authors. Several solutions to eliminate artifacts are presented comprehensibly. Two chapters on clinical decision-making algorithms describe a useful approach to collecting significant information from monitors and how to use this information to improve patient care and outcome. The section ends with an overview of the economic issues involved in intensive care technology. The critical care health system now costs an estimated $\$ 100$ billion/year in the United States. New technology should not be developed solely for the spirit of innovation but should improve patient outcome in a cost-effective manner. The information provided in this chapter is useful for physician intensivists, who design studies of administrative decisions, quality assurance, and budget planning.

The second section, respiratory monitoring, begins with a detailed discussion of respiratory monitoring and gadgets. The opening chapter is dedicted to the clinical assessment of the respiratory system, a recurrent approach in the book where clinical assessment of organ function always precedes the technical aspects of monitoring. The respiratory system is the most comprehensively reviewed. The dynamics of gas exchange and oxygen transport are analyzed in detail. Tables and diagrams are well selected from several physiology texts and review articles. Interestingly, this section not only illustrates new technology but critically analyzes the inaccurancy of potentially promising monitoring systems. One example, continuous intravascular blood gas and $\mathrm{pH}$ monitoring, a promising and potentially useful technology, never obtained widespread use due to the erratic values obtained from partial obstruction of the arterial catheter.

The second section also extensively reviews oxygen monitoring and includes pulse oximetry, transcuteneous and trans-conjunctival oxygen analyzers, and mixed venous oxygen monitoring. The review is accurate, complete, and informative. This section ends with a review of the mechanisms that control breathing and the pathophysiology of respiratory muscle dysfunction. It includes a review of most noninvasive monitoring tools available for the bedside evaluation of respiratory function and respiratory mechanics, which helps the reader understand the dynamics of weaning ventilatordependent patients. The oxygen monitoring section is the most informative part of the book, with many selfexplanatory pictures and diagrams, which have been edited and improved since original publication. The reference literature is well selected and updated to 1997.

The third section reviews the cardiovascular system. Although comprehensive and informative, we would have liked additional clinical case presentations, diagrams, and tables.

In the review of arterial pressure recording devices and pulmonary artery catheters, the author reviewed in detail all the factors contributing to false interpretation of hemodynamic data. The chapter on transesophageal echocardiography is appreciated for the richness of color pictures. A review of hemodynamic interpretation and treatment algorithms would have been useful. It is possible that with the development of smaller size probes, transesophageal echocardiography can provide a 24hour display of ventricular performance, which would represent a major breakthrough in ICU patient care.

The fourth section on neurological monitoring reviews the latest technology, including cerrebral oximetry and transcranial Doppler application. However, the section on electroencephalography in the ICU should have included a description of bispectral electroencephalogram analysis. This device, recently released on the market, can be potentially useful in the ICU.

In section six, we found particularly useful a review of patient monitoring during transportation. A performance comparison of available transportable monitoring devices, such as ventilators and hemodynamic monitors, pacemakers, and defibrillators, would have been very useful to the ICU practitioner who might wish to purchase a transportable device for his unit. The logistics of point-of-care testing in the ICU are extensively reviewed and very informative. The strategic placement of point-of-care is as important as the presence of competent medical personnel. An efficient communications network and laboratory data analysis are key elements in a modern ICU. 
The last section assesses standard of care and includes monitoring standards, level of monitoring intensity, safety issues in monitoring, and basic ethical and legal issues, such as discontinuation of life support, testing new technology in the ICU, and physician's relationship with the Food and Drug Administration.

A short appendix on selection of monitoring equipment lists the major devices, their manufacturer, and a range of prices, updated to the year 1997. This is a useful reference for medical personnel and administrators involved in building or remodeling an ICU.

In conclusion, Dr. Tobin has edited a textbook that addresses the available technology in the ICU, and provides useful guidelines for correct interpretation of data in difficult clinical conditions. This is a rapidly expanding technology that requires continuous updates. This is a Must-Have reference book for every critical care physician, ICU health care provider, and technical support personnel.

Andrea Gabrielli, MD

Lawrence J. Caruso, MD

Department of Anesthesiology

UF/College of Medicine

Gainesville, FL 32610, U.S.A. 